\title{
Rappel: le gel de l'ouverture de nouveaux cabinets est prolongé - il faut agir rapidement!
}

Urs Reinhard, lic. en droit, avocat, bureau juridique de l'AMPHS*

Hanspeter Kuhn, avocat, secrétaire général adjoint de la FMH

\footnotetext{
* Adresse et heures de réception: Association des médecins principaux d'hôpitaux de Suisse AMPHS, Gutenbergstrasse 9, 3011 Berne, tél. 03139025 65, fax 03139025 64, e-mail: vlss@hin.ch. Lundi au vendredi $8-12 \mathrm{~h}$ et $13 \mathrm{~h} 30-17 \mathrm{~h} 30$. leurs factures de façon autonome et n'utilisent donc pas leur numéro RCC personnel bien qu'étant au bénéfice du droit de liquidation, ont avantage à s'annoncer auprès de la direction des affaires sanitaires de leur canton avant la fin de l'année.
}

Correspondance:

$\mathrm{FMH}$

Service de droit

Elfenstrassee 18

CH-3000 Berne 15

Tél. 0313591111

Fax 0313591112

E-mail: lex@fmh.ch

\section{Médecins salariés exerçant dans les soins ambulatoires (HMO, etc.)}

$K n$. Pour les médecins salariés dans le domaine ambulatoire aussi, la question de savoir si, aux yeux de la direction des affaires sanitaires de leur

\section{Médecins-chefs et médecins adjoints}

Re. La prolongation décidée par le Conseil fédéral du gel de l'ouverture des cabinets médicaux est en vigueur depuis le 4 juillet 2005. Selon cette nouvelle réglementation les autorisations de pratiquer déjà octroyées expirent si l'on en fait pas usage dans un laps de temps déterminé [1]. Ce délai est de 6 mois, mais peut être prolongé par les cantons à 12 mois [2]. Si aucune prolongation n'a été décidée, le jour fixé pour l'expiration est donc le 3 janvier 2006. Les personnes qui n'ont pas (encore) fait usage de cette prolongation doivent donc agir rapidement.

Le Bulletin des médecins suisses (BMS) a déjà publié de nombreux articles sur la manière de s'opposer à la perte de l'admission à pratiquer [3] . Il convient cependant de souligner une fois de plus que s'il reste des points non éclaircis, il faut en discuter avec le canton. Les médecins adjoints et les médecins-chefs qui n'établissent pas (ou ne peuvent établir), pour une raison ou une autre, canton, ils sont considérés comme des médecins en pratique privée ou non, est importante. Il semblerait que ces derniers temps les choses bougent dans les cantons et chez santésuisse à ce propos [4]. Des informations complémentaires paraîtront dès que possible dans le Bulletin des médecins suisses.

\section{Aperçu de la page interne de I'ASMAC - cantons qui n'ont pas prolongé le délai}

$K n$. L'ASMAC établit sur son site l'aperçu des cantons: www.vsao.ch $\rightarrow$ Association ASMAC $\rightarrow$ actualités $\rightarrow$ blocage de l'accès à la pratique privée. Selon l'aperçu de l'ASMAC, les cantons suivants n'ont pas prolongé le délai, ce qui le rend effectif depuis le 3 janvier 2006: Appenzell Rhodes-intérieures; Bâle-Campagne; Berne; Genève (ne vaut cependant pas pour les autorisations selon l'ancien droit); Nidwald; Obwald; Schaffhouse; Tessin; Uri.

\section{Références}

1 Art. 55a, al. 4 LAMal: «L'admission, une fois délivrée, expire lorsqu'il n'en est pas fait usage pendant un certain délai. Le Conseil fédéral fixe les conditions.»

2 Art. 3a. al. 1 et art. 3, al. 2 et 3 LAMal.

3 Voir les articles de Hanspeter Kuhn dans Bull Méd Suisses 2005;86(23):1392-4 et 2005; 86(29/30):1780-4 et 1784-7.

4 Information de décembre 2005 de Daniel Staffelbach, avocat (Walder Wyss \& Partner, Münstergasse 2, 8001 Zurich) adressée à HP Kuhn. 